\title{
Cryptococcemia resulting in an incomplete abortion in an HIV-positive patient
}

\author{
Kurosh Rahimi MB ChB FRCPC, Runjan Chetty MBBCh FRCPC DPhil, Blaise Clarke MB ChB FRCPC
}

\begin{abstract}
K Rahimi, R Chetty, B Clarke. Cryptococcemia resulting in an incomplete abortion in an HIV-positive patient. Can J Infect Dis Med Microbiol 2009;20(3):e97-e99.

Exotic infections and unusual presentations are the norm in immunocompromised patients. An unusual case of cryptococcemia resulting in a miscarriage is reported. This was the first presentation of the disease and the sentinel infection in the patient who was found to be HIV positive. Histological evaluation of the dilation and curettage specimen showed numerous typical organisms of cryptococcus present within the products of conception. There was a focal granulomatous response to the organisms. An accompanying cryptococcal endometritis was not noted. Other concomitant infections were not seen.
\end{abstract}

\section{Cryptococcémie à l'origine d'un avortement incomplet chez une patiente VIH positive}

Key Words: Cryptococcus; HIV; Incomplete abortion

$\mathrm{C}$ ryptococcus is an encapsulated yeast that causes opportunistic infection in immunocompromised patients, involving typically the nervous and respiratory systems. Traditionally, risk groups have included patients with diabetes, renal failure or cirrhosis, patients with connective tissue disease, patients on steroids or monoclonal antibody therapy, solid organ transplant recipients, patients with hematological malignancies and pregnant women. The HIV epidemic has facilitated an increase in the incidence of this infection, with HIV coinfection implicated in $80 \%$ of cases of cryptococcosis worldwide (1). In addition, HIV infection often results in unusual clinical presentations of well-known organisms such as cryptococcus.

Cryptococcosis of the female genital tract is rare, with descriptions of involvement of the endometrium, ovary, vagina, uterus and placenta confined to case reports. In the present report, we document the presence of cryptococcal organisms in the dilation and curettage specimen of an incomplete miscarriage in an HIV-positive woman. This is the third case of cryptococcus involving the female genital tract in pregnancy and is noteworthy because it was the initial presentation of the infection, resulting from presumed cryptococcemia.

\section{CASE PRESENTATION}

A 28-year-old African patient (para 2, gravida 3) presented to the emergency department complaining of acute abdominal pain and vaginal bleeding. On examination, the patient appeared to be in her first trimester, was shocked, there were no signs of fetal life and a dilation and curettage was performed to evacuate the pregnancy. Despite this intervention the patient died shortly thereafter. The patient did not have any antenatal records and presented for the first time as a medical emergency.

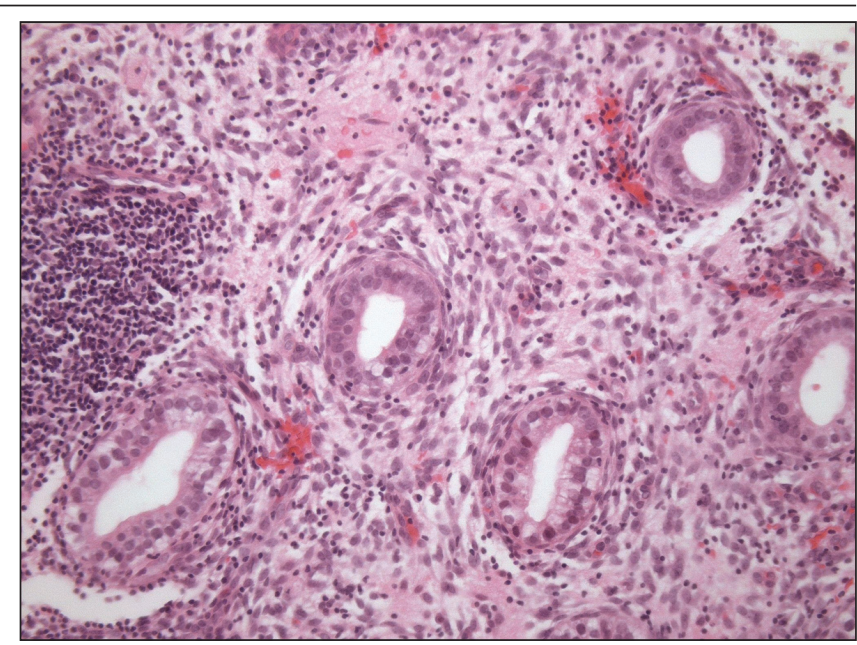

Figure 1) Endometrium with predominantly chronic inflammation featuring frequent plasma cells. Cryptococcal organisms are not seen

An autopsy was not performed. In addition, because of the immediate demise of the patient, a microbiological culture was not requested. As part of the initial work-up of the patient, blood was taken for HIV, which was positive.

\section{Pathology}

The dilation and curettage specimen contained blood and fibrin with endometrial tissue fragments showing stromal decidualization and mixed, but predominantly chronic, inflammation (Figure 1). Focal cyto- and syncytiotrophoblasts were present admixed with hemorrhage (Figure 2). Encapsulated organisms consistent with cryptococcus were noted lying singly and in

Department of Pathology, University Health Network/University of Toronto, Toronto, Ontario

Correspondence: Dr Runjan Chetty, Department of Pathology, University Health Network/Toronto Medical Laboratories, The Toronto General

Hospital, 200 Elizabeth Street, 11th Floor, Eaton Wing, Toronto, Ontario M5G 2C4. Telephone 416-340-3661, fax 416-340-5517,

e-mail runjan.chetty@uhn.on.ca 


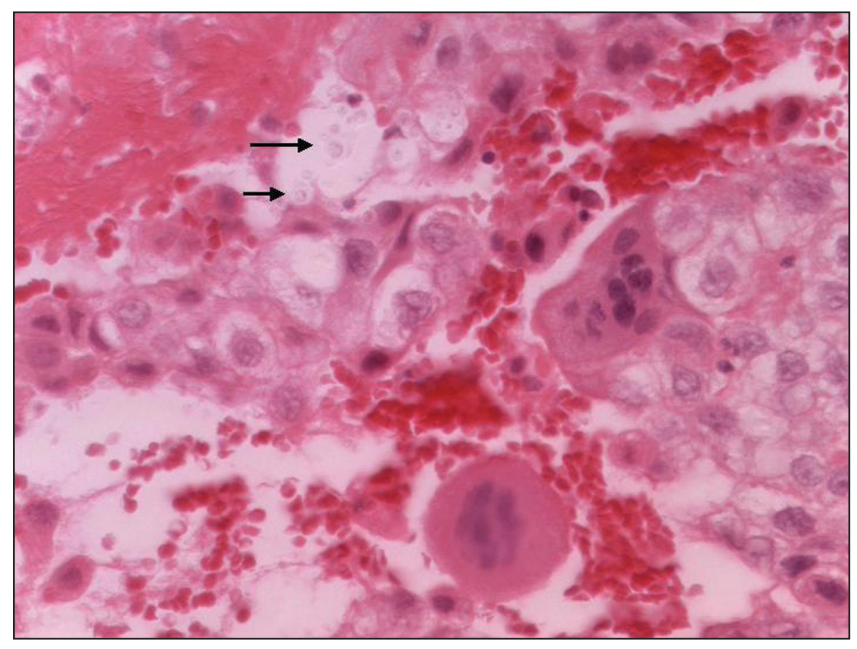

Figure 2) Trophoblasts admixed with fibrin and hemorrhage with several of fungal organisms of cryptococcus (arrows)

clusters enmeshed in the fibrin and hemorrhage with an associated granulomatous response (Figure 3). There were no organisms present in the endometrial tissue. A mucin stain highlighted the capsule of the organisms. Other fungi or tuberculosis were not seen on special stains.

\section{DISCUSSION}

Thus far, there have been two reports of cryptococcal placentitis. The initial case, reported by Kida et al (2), was of disseminated cryptococcosis in a HIV-positive patient with the subsequent case by Molnar-Nadasdy et al (3) occurring in a patient with systemic lupus erythematosus.

Both cases documented intervillous (maternal) space involvement by cryptococcal organisms associated with fibrin and neutrophils. Both specifically report the absence of chorioamnionitis, villitis or organisms within the villi (fetal compartment). In the case reported by Kida et al, the woman died on postpartum day 2. Postmortem examination revealed disseminated cryptococcus involving the lungs, liver and kidney but there was no documentation of uterine involvement. The infant was well and several months after birth had no clinical evidence of HIV or cryptococcal infection.

In the second case, the neonate died on day 5 but an autopsy was not performed. The mother did well. Having undergone a caesarean hysterectomy, histological examination of the uterus revealed no evidence of deciduitis and again, no organisms within the endometrium.

In our case, the yeasts were identified within the fibrin but did not involve the endometrium. This in conjunction with the other two reports documenting involvement of the maternal (intervillous space) would suggest hematogenous dissemination of the organism.

In the veterinary literature, Petrites-Murphy et al (4) documented a case of equine cryptococcal endometritis and placentitis in which the foal died on day 9 due to cryptococcal pneumonia. In this case, the endometrium contained clusters of giant cells some of which contained cryptococcal organisms. Cryptococcal endometritis was not seen in the current case or in the two previous reports of cryptococcal placentitis.

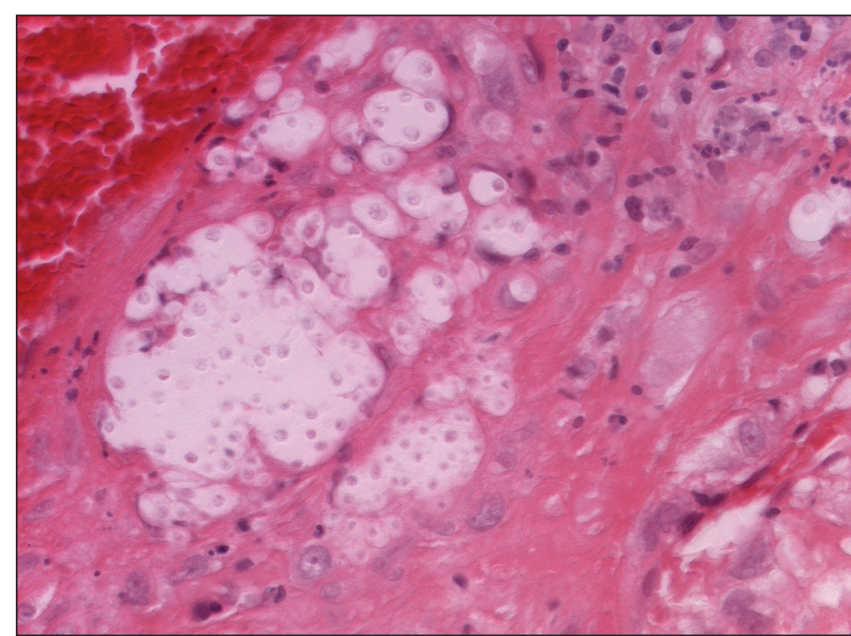

Figure 3) Under higher magnification, numerous cryptococcal organisms admixed with fibrin and inflammation can be appreciated

Plaut (5) reported a case of cryptococcosis involving the cervix, endometrium and fallopian tube of a 31-year-old woman. Granulomatous lesions with giant cells containing fungal organisms were noted on microscopy, and on microbiological culture the organism was interpreted as being consistent with Cryptococcus glabratus.

Although it is recognized that cryptococcus can affect pregnant women, it is still controversial whether there is motherto-child transmission. Sirinavin et al (6) reported a case of presumed mother-to-child transmission of cryptococcus, in which Cryptococcus neoformans was present in the cerebrospinal fluid of an infant at 92 days of age (6). They postulate it was acquired during labour or delivery basing this assumption on the fact that the mother had peripartum cryptococcosis and disease onset in the child was before three months of age.

Cryptococcal infection may be protean in its manifestations and this is particularly true in the setting of immune compromise, such as HIV. Even in the absence of immune compromise, unusual presentations have been documented. Chen et al (7) documented a case of isolated vaginal cryptococcal infection in an elderly patient treated for colon cancer nine years previously. The current case and the previous report of cryptococcal placentitis occurred in the setting of HIV infection. In addition, a case of cryptococcoma presenting as a tubo-ovarian abscess in an HIV-positive patient has recently been reported (8).

Pregnancy itself results in altered immune state possibly attributable to increased suppressor $\mathrm{T}$ cell function, decreased CD4 $\mathrm{T}$ cell function and stimulation and decreased production of cytokines. Disseminated fungal infections, which have been described in pregnancy in otherwise healthy women, include coccidioidomycosis, African histoplasmosis and blastomycosis.

Several other fungi involving the placenta have been described in otherwise healthy pregnant women and in women with secondary causes of immune compromises. These organisms include histoplasma, candida, Coccidioides immitis and blastomyces.

Whitt et al (9) documented the first cases of transplacental transmission of Histoplasma capsulatum in humans, having previously only been diagnosed in horses. The case was of a woman with AIDS with disseminated histoplasmosis in whom 
blood cultures were positive for $H$ capsulatum. Culture of infant blood, cerebrospinal fluid and peritoneal fluid also grew $\mathrm{H}$ capsulatum. Histological examination of the placenta showed focal yeast containing histiocytes within stem villi and trophoblasts but not within the intervillous space. The umbilical cord and membranes showed absence of chorioamnionitis and vasculitis.

Lemos et al (10) reported three cases of blastomycosis in pregnancy and reviewed a further 16 cases from the literature. Overall the placenta was examined in only seven cases, six of which were negative for fungal organisms. In the one documented case of blastomycosis placentitis, the neonate was healthy.

Arnold et al (11) recently presented the third case of pregnancy-associated disseminated coccidioidomycosis in which the diagnosis was determined by histological examination of the placenta. Typical histological features include areas of necrotic villi, granulomatous reaction with giant cells, microabscesses and endospores enmeshed in fibrin. Other findings include coagulative necrosis of the villi, chronic chorionic plate vasculitis and acute and chronic villitis. It is thought that coccidioides does not cross the placenta because there are numerous reports of placentitis without fetal disease. Postulated reasons for this include the large size of the organism and containment by the granulomatous inflammatory reaction. Coccidioidomycosis is rare in infants, although some authors speculate that neonatal infection may occur in vaginal delivery because of postnatal cervical cultures positive for $\mathrm{C}$ immitis.

Fitzhugh et al (12) described a case of a premature infant in which placental examination revealed acute chorioamnionitis and organisms morphologically consistent with Actinomyces.

Causes of chronic endometritis of specific type include other fungal organisms, bacteria and viruses. Herpes simplex virus, cytomegalovirus (CMV) and HIV may infect the endometrium.

Rare infective causes of miscarriage that have been documented include syphilitic endometritis and CMV endometritis. Lee et al (13) documented a case of syphilitic endometritis causing first trimester miscarriage in 30-year-old. Examination of the dilation and curettage specimen showed lymphoplasmacytic deciduitis and decidual necrosis with silver staining highlighting spirochetes diffusely involving endometrial glands and stroma.

Dehner et al (14) have reported a case of CMV endometritis associated with spontaneous abortion, and work by Kleinman et al (15) showing estradiol induced reactivation of CMV in endometrial cells implicates the hormonal milieu of pregnancy in viral reactivation.

Placental changes in HIV are not well documented. In a prospective study of 51 placenta from treated and untreated HIV-positive women, chorioamnionitis $(31.37 \%)$ and deciduitis $(21.56 \%)$ were the most common inflammatory findings with noninflammatory lesions including cytotrophoblastic hyperplasia $(76.47 \%)$, subchorionic fibrin $(52.94 \%)$, calcification $(49.01 \%)$, perivillous fibrin $(43.13 \%)$ and chorangiosis (29.4\%) (16). Villitis has generally been reported to be rare in HIV-related pregnancies, and because this is an immunological phenomenon, this has been attributed to the lymphopenia.

\section{CONCLUSION}

We present an unusual case of cryptococcemia-induced incomplete abortion in an HIV-positive patient as the initial presentation of this infection. This is the first documented case of cryptococcal-associated miscarriage and it expands the spectrum of HIV-related disease in pregnancy.

\section{REFERENCES}

1. Chayakulkeeree M, Perfect JR. Cryptococcosis. Infect Dis Clin North Am 2006;20:507-44.

2. Kida M, Abramowsky CR, Santoscoy C. Cryptococcosis of the placenta in a woman with acquired immunodeficiency syndrome. Hum Pathol 1989;20:920-1.

3. Molnar-Nadasdy G, Haesly I, Reed J, et al. Placental cryptococcosis in a mother with systemic lupus erythematosus. Arch Pathol Lab Med 1994;118:757-9.

4. Petrites-Murphy MB, Robbins LA, Donahue JM, et al. Equine cryptococcal endometritis and placentitis with neonatal cryptococcal pneumonia. J Vet Diagn Invest 1996;8:383-6.

5. Plaut A, Human infection with Cryptococcus glabratus; report of case involving uterus and fallopian tube. Am J Clin Pathol 1950;20:377-80.

6. Sirinavin S, Intusoma U, Tuntirungsee S. Mother-to-child transmission of cryptococcus neoformans. Pediatr Infect Dis J 2004;23:278-9.

7. Chen CK, Chang DY, Chang SC, et al. Cryptococcal infection of the vagina. Obstet Gynecol 1993;81:867-9.

8. Sing Y, Ramdial PK, Ibrahim T. Cryptococcosis masquerading as a tuboovarian abscess. Int J Gynecol Pathol 2008;27:37-40.

9. Whitt SP, Koch GA, Fender B, et al. Histoplasmosis in pregnancy:

Case series and report of transplacental transmission. Arch Intern Med 2004;164:454-8.

10. Lemos LB, Soofi M, Amir E. Blastomycosis and pregnancy. Ann Diagn Pathol 2002;6:211-5.

11. Arnold CA, Rakheja D, Arnold MA, et al. Unsuspected, disseminated coccidioidomycosis without maternofetal morbidity diagnosed by placental examination: Case report and review of the literature. Clin Infect Dis 2008;46:e119-123.

12. Fitzhugh VA, Pompeo L, Heller DS. Placental invasion by actinomyces resulting in preterm delivery: A case report. J Reprod Med 2008;53:302-4.

13. Lee WK, Schwartz DA, Rice RJ, et al. Syphilitic endometritis causing first trimester abortion: A potential infectious cause of fetal morbidity in early gestation. South Med J 1994;87:1259-61.

14. Dehner LP, Askin FB. Cytomegalovirus endometritis: Report of a case associated with spontaneous abortion. Obstet Gynecol 1975;45:211-4.

15. Kleinman D, Sarov I, Insler V. Reactivation of cytomegalovirus in endometrial cells by estradiol. Gynecol Obstet Invest 1986;21:136-43.

16. D'Costa G F, Khadke K, Patil YV. Pathology of placenta in HIV infection. Indian J Pathol Microbiol 2007;50:515-9. 


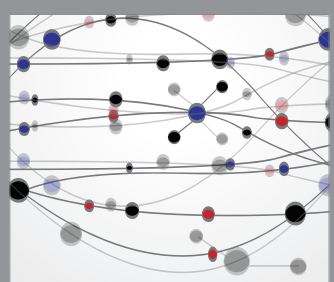

The Scientific World Journal
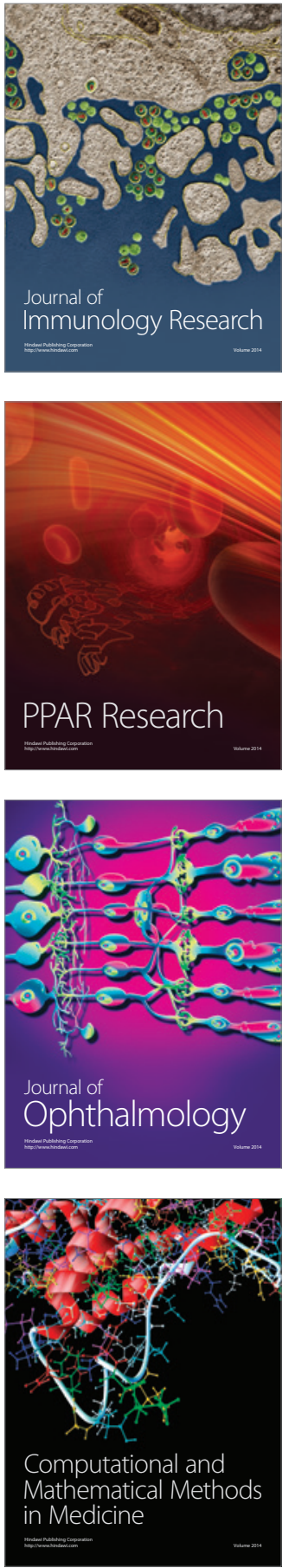

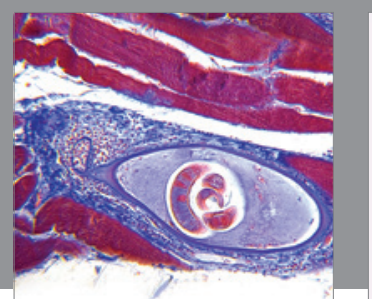

Gastroenterology Research and Practice

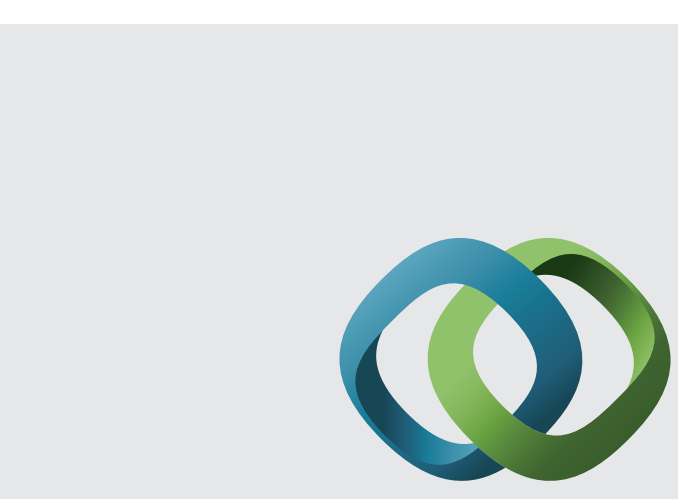

\section{Hindawi}

Submit your manuscripts at

http://www.hindawi.com
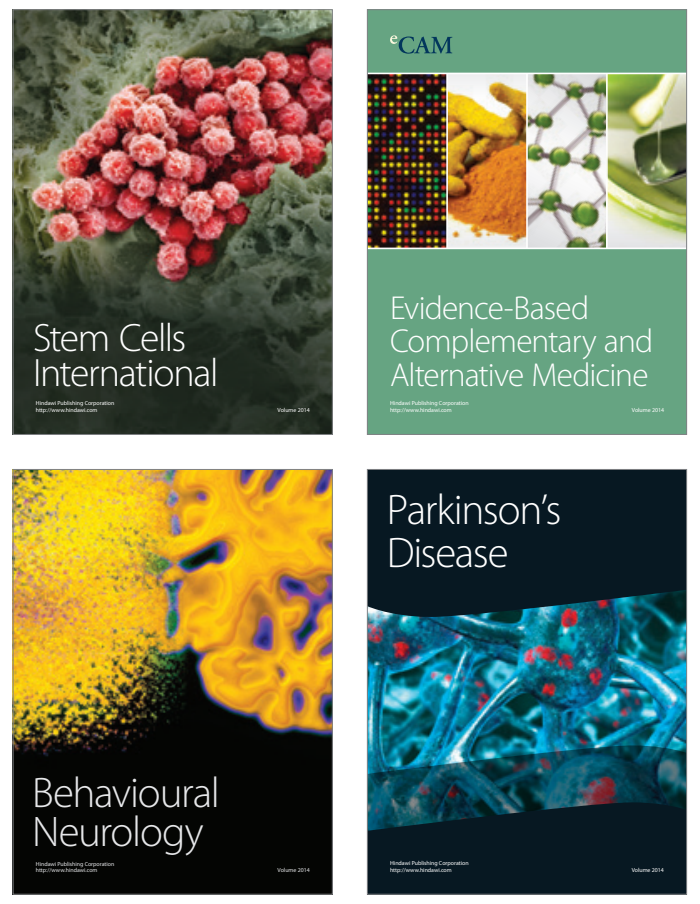
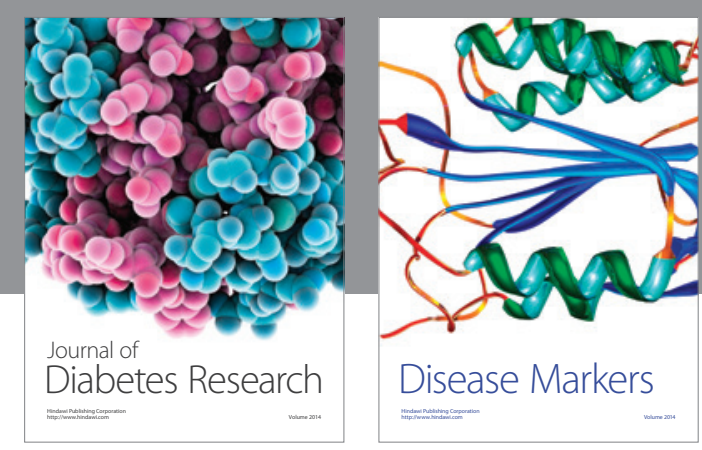

Disease Markers
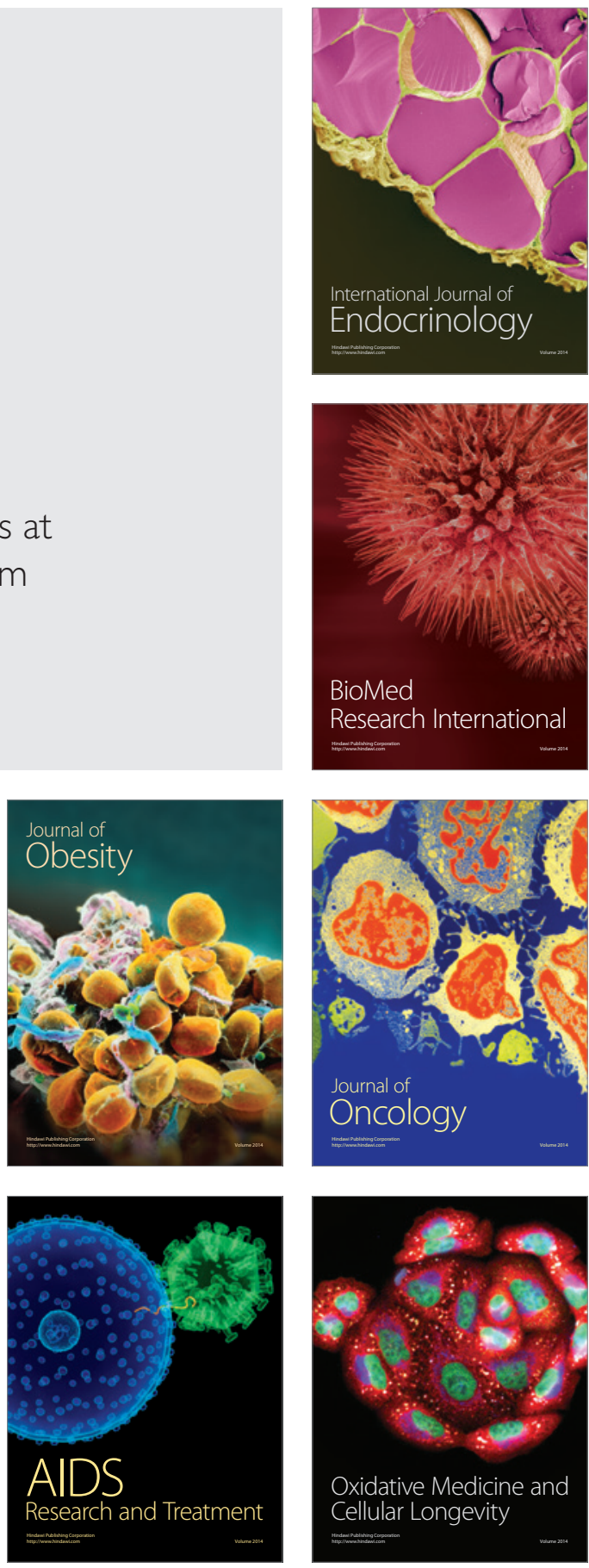